\title{
INFLUENCE OF SOIL AND TOPOGRAPHY ON THE COMPOSITION OF A TREE COMMUNITY IN A CENTRAL BRAZILIAN VALLEY FOREST
}

\author{
J. R. R. Pinto ${ }^{1}, A \cdot T \cdot$ Oliveira-Filho ${ }^{2} \&$ J. D. V. Hay
}

The purpose of the present study was to investigate the correlations between environmental variables related to the substrate (soil and topography) and the distribution of tree species in a valley forest in the Chapada dos Guimarães National Park, Mato Grosso State, Brazil. The forest lies in the Cerrado Domain of Central Brazil where the dominant vegetation cover is cerrado (woody savanna). A survey of the tree community registered all live individuals with circumference at breast height $(\mathrm{cbh}) \geq 15 \mathrm{~cm}$ found in eighteen $600 \mathrm{~m}^{2}$ plots (total area $1.08 \mathrm{ha}$ ). The substrate variables used in the gradient analyses were obtained from a topographic survey and from analyses of the chemical and physical properties of soil samples. A principal components analysis of soil and topography variables and a canonical correspondence analysis of the species-environment relationships produced similar results, separating both the substrate variables and the tree species abundances, mainly according to the two types of bedrock, sandstone or slate, underlying their soils, and secondly to the three topographic sectors recognized: Streamside, Mid Slope and Upper Slope. The differences in soil fertility and texture (related to the bedrocks) and the soil water regime (related to both soil texture and topography) were probably the chief factors determining the distribution of tree species in the forest.

Keywords. Brazil, Cerrado Domain, Chapada dos Guimarães, multivariate analysis, soil catena, tree species distribution, valley forest.

\section{INTRODUCTION}

Of the world's ecosystems, tropical forests are presently attracting much attention from ecologists, not only because of their species diversity but also because of their composition and complex structure. One of the components of this complexity is the heterogeneity of habitats, which influences the spatial distribution of plant species. Spatial heterogeneity in the physical environment is an important factor contributing to the commonly high tree species diversity of tropical forests, as variations in resource availability in both horizontal (particularly soil chemical and physical properties, and ground-water regime) and vertical (canopy layering, rooting zones) directions allow niche differentiation among tree species (Fowler, 1988; Terborgh,

\footnotetext{
${ }^{1}$ Departamento de Engenharia Florestal, Universidade de Brasília, CP 04357, CEP 70919-970, Brasília, DF, Brazil.

${ }^{2}$ Corresponding author. Departamento de Ciências Florestais, Universidade Federal de Lavras, CP 37 , CEP 37200-000, Lavras, MG, Brazil. E-mail: ary@ufla.br

${ }^{3}$ Departamento de Ecologia, Universidade de Brasília, CP 04631, CEP 70919-970, Brasília, DF, Brazil.
} 
1992). Development of the gap-phase dynamics theory for tropical forests (see Denslow, 1987 for a review) has made it clear that temporal heterogeneity of the environment (disturbance history) also plays a very important role in determining tree species distribution. Unfortunately, these findings have also played down, to a considerable extent, the notion that the substrate plays the major role in determining the horizontal patterns of tree species distribution (Clark et al., 1998).

Tropical riverine forests are typical examples of high environmental-vegetational heterogeneity occurring over relatively small areas caused mainly by strong variation in the substrate, particularly ground-water regime and soil chemical and physical properties (Oliveira-Filho et al., 1994; Metzger et al., 1997; Hall \& Harcombe, 1998; van den Berg \& Oliveira-Filho, 1999; Rodrigues \& Nave, 2000; Rosales et al., 2001; Botrel et al., 2002). Although cerrado (woody savanna) is the dominant vegetation of the Cerrado Domain in Central Brazil, riverine forests are a very common element of the landscape because forests fringe nearly all water-bodies in the region. This forest network depends on high soil moisture throughout the year providing a suitable habitat for a large number of typical moist forest species despite the long dry season of the region (Oliveira-Filho \& Ratter, 1995, 2002). Most of the striking variation of riverine forests in Central Brazil, in both physiognomy and floristic composition, results from variation in topography and drainage, together with soil properties (Oliveira-Filho et al., 1990, 2001; Felfili, 1995; Silva Júnior et al., 1996; Schiavini, 1997; Ribeiro \& Walter, 1998; Rodrigues \& Shepherd, 2000; Marimon et al., 2002).

In some steep valleys of Central Brazil, riverine forests lie alongside mesophytic forests (instead of cerrado or grasslands) making up the so-called valley forests (Eiten, 1983, 1994; Oliveira-Filho \& Ratter, 2002), many of which are sheltered in canyons and flanked by escarpments of sandstone plateaux. Since the descriptive accounts of Askew et al. (1971), Ratter et al. (1973, 1978) and Eiten (1975), Central Brazilian valley forests have received little attention, and, to our knowledge, there are no studies on their vegetation-environment relationships. In this paper we assess the correlations between environmental variables related to the substrate (soils and topography) and the distribution of tree species in a valley forest of the Chapada dos Guimarães. Our initial hypothesis was that variations in tree species abundances were chiefly related to elevation above the valley bottom.

\section{Materials and Methods}

\section{The study area}

The study area is a valley forest in the Chapada dos Guimarães National Park (PNCG), Mato Grosso State, Brazil, that lies at the bottom of the canyon below the Véu de Noiva ('Bridal Veil') waterfall, on the Coxipozinho stream at $15^{\circ} 24^{\prime} 25^{\prime \prime} \mathrm{S}$ and $55^{\circ} 50^{\prime} 25^{\prime \prime} \mathrm{W}$ (Fig. 1). The scenic beauty of this area makes the Véu de Noiva waterfall the second most visited attraction of the Park (Macedo \& Pinto, 1996). 


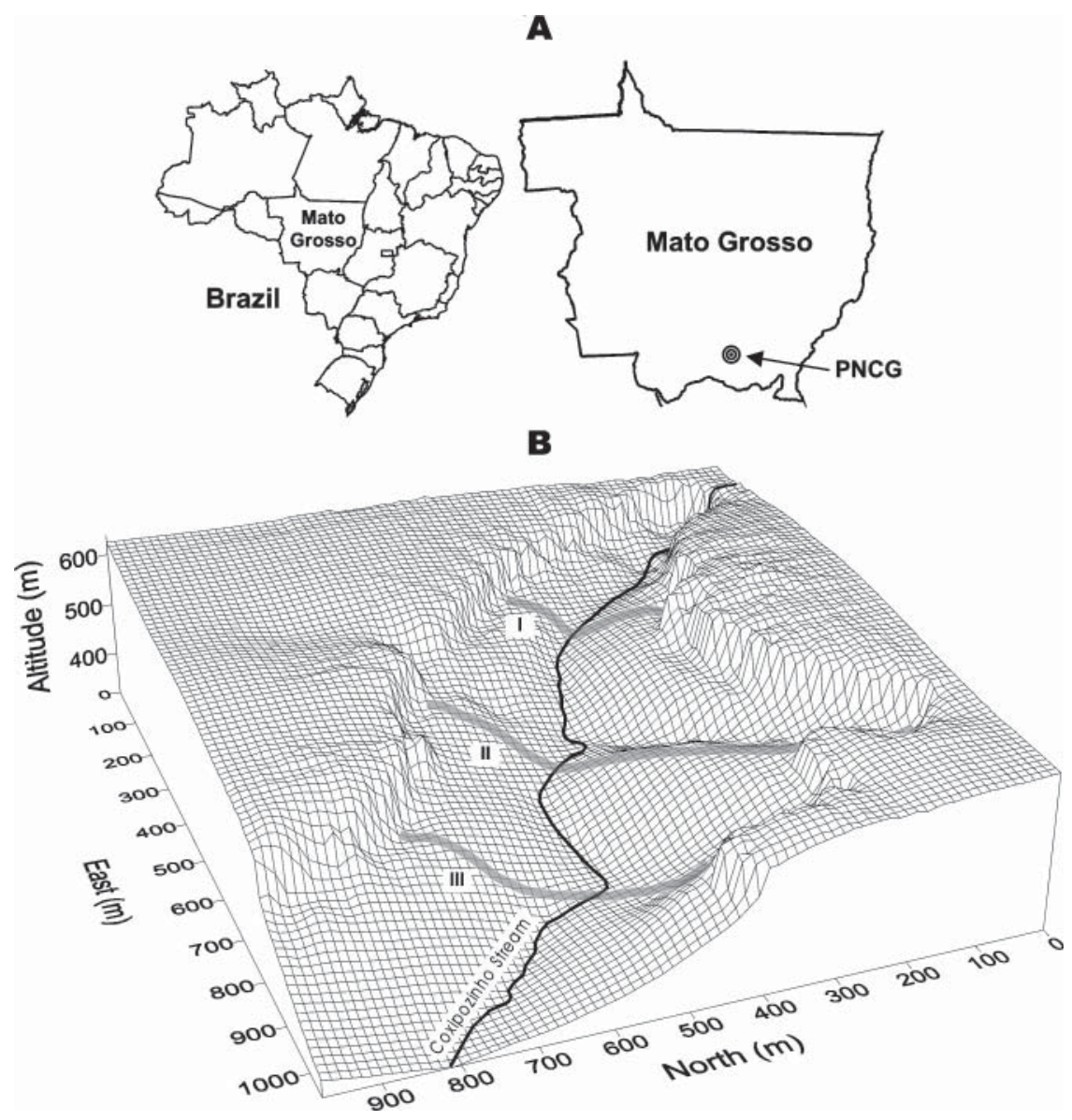

FIg. 1. A, Position of the Chapada dos Guimarães National Park (PNCG) in Mato Grosso State, Brazil. B, Surface grid of the canyon of the Véu de Noiva waterfall showing positions of the three sample transects (I, II, III). Lines on the surface grid are at $12.5 \mathrm{~m}$ intervals.

Altitudes range from $300-800 \mathrm{~m}$ in the Park, and from $340-610 \mathrm{~m}$ in the canyon. The regional climate is classified as Köppens Aw and Cw types (IBAMA, 1994), both characterized by a dry winter, from May to September, and a rainy summer, from October to March. The mean annual precipitation ranges from 1800-2000mm (Oliveira-Filho \& Martins, 1986). The Chapada dos Guimarães plateau is formed of Devonian sandstones and rests on the Pre-Cambrian slates that predominate in the surrounding lowlands, the Baixada Cuiabana (Oliveira-Filho \& Martins, 1986, 1991). The predominant soils in the region are dystrophic quartzitic sands at the top and the base of the plateau, dystrophic red-yellow latosols (oxysols) on the lowlands, and litholic soils formed over either sandstone or slate (MMA, 1982). Most soils in the forest studied are shallow; rocky outcrops are frequent and slopes are steep $\left(20-50^{\circ}\right)$; sandstone bedrock is replaced by slate at lower elevations. The predominant vegetation cover of the Park is cerrado, with its wide array of 
physiognomic forms; seasonal marshes are found where the water table is periodically near or at the surface; forests occur as narrow galleries, valley forests, or small patches of semideciduous forest (IBAMA, 1994).

\section{Survey of the tree community and environmental variables}

We surveyed the tree community in 18 permanent plots placed on catena transects aligned at right angles to the line of the watercourse and crossing both slopes to end at the foot of the escarpments on each side. We placed the transects in three sites along the canyon: Transect I at the inner end near the waterfall, Transect II in the middle and Transect III near the mouth (Fig. 1). We marked out a total of 18 plots of $600 \mathrm{~m}^{2}$ (total area $1.08 \mathrm{ha}$ ) at three topographic sectors in each of the six slopes crossed by the transects: Streamside, Mid Slope and Upper Slope. Plot dimensions were $10 \mathrm{~m} \times 60 \mathrm{~m}$ in the Streamside sectors and $20 \mathrm{~m} \times 30 \mathrm{~m}$ in the Mid Slope and Upper Slope; the shortest side was aligned parallel to the transect. We identified to species level all live trees in the plots with a circumference at breast height $(\mathrm{cbh}) \geq 15.0 \mathrm{~cm}$, measuring this with a tape and estimating total height with the help of a pole.

We carried out a topographic survey along each of the three transects and the watercourse, and prepared a contour map and associated surface grid. Following the method used by van den Berg \& Oliveira-Filho (1999), we calculated for each plot the topographic variable 'vertical distance to the watercourse' from the difference in elevation between the plot centre and the valley bottom at the transect crossing. We collected four 0.5 litre soil samples from depths of $0-20 \mathrm{~cm}$ at the midpoints between the corners and at the centre of each plot. The five soil samples were bulked and subsampled for each plot. Chemical and physical analyses were carried out at the Soil Laboratory of the Universidade Federal de Lavras following the procedures proposed by EMBRAPA (1997).

\section{Environmental analysis and tree community descriptive variables}

To identify the main variation patterns of the substrate in the area we performed a principal components analysis, PCA (Kent \& Coker, 1992), of topography and soil variables using CANOCO 4 for Windows (ter Braak \& Šmilauer, 1998). The variables included: soil physical properties (proportions of sand, silt and clay), soil chemical properties ( $\mathrm{pH}$, extractable $\mathrm{P}$, exchangeable bases $\left[\mathrm{K}^{+}, \mathrm{Ca}^{2+}, \mathrm{Mg}^{2+}\right.$ and $\left.\mathrm{Al}^{3+}\right]$ and sum of bases $\left[\mathrm{SB}=\mathrm{K}^{+}+\mathrm{Ca}^{2+}+\mathrm{Mg}^{2+}\right]$ ), proportion of soil organic matter, and vertical distance to the watercourse. Variables expressed as proportions were previously transformed by the expression $\arcsin \left(x^{1 / 2}\right)$.

The pattern arising from the PCA indicated two large groups of plots separated essentially by soil fertility and texture, reflecting whether the local bedrock was sandstone or slate. These two groups could be further split into five types of site based on the topographic sectors (Fig. 2), grouped as follows: 

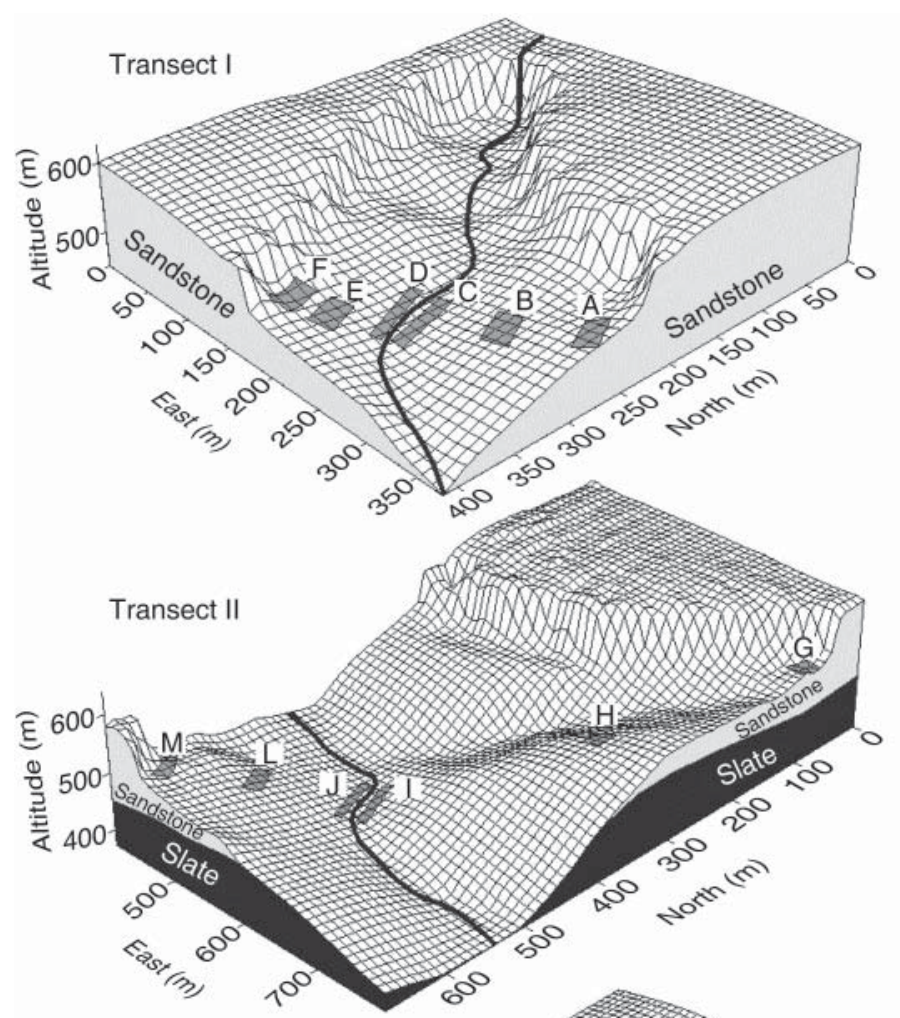

Transect III

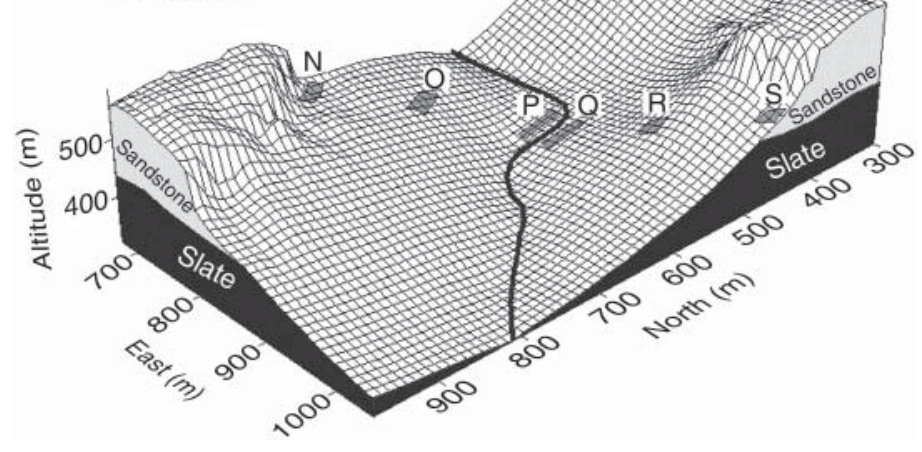

FIG. 2. Surface grids of sections of the canyon of the Véu de Noiva waterfall at the three sample transects showing the distribution of plots and the underlying bedrock. Lines on the surface grid are at $10 \mathrm{~m}$ intervals.

- Sandstone/Upper Slope (plots A, F, G, M, N, S)

- Sandstone/Mid Slope (plots B, E, H, L)

- Sandstone/Streamside (plots C, D)

- Slate/Mid Slope (plots O, R)

- Slate/Streamside (plots I, J, P, Q). 
We compared soil variables among the five sites with analyses of variance, applying Tukey-Kramer tests to variables with significant differences (Zar, 1996). Variables expressed as proportions were previously transformed by the expression $\arcsin \left(x^{1 / 2}\right)$.

\section{Species-environment relationship}

We performed a canonical correspondence analysis, CCA (ter Braak, 1987, 1995), to investigate the relationships between species abundance and environmental (substrate) variables in the 18 plots using CANOCO 4 for Windows (ter Braak \& Šmilauer, 1998). The species abundance matrix consisted of the number of trees per plot, but included only the 39 species with 10 or more trees in the total sample. As recommended by ter Braak (1995), all abundance values were log-transformed before being analysed, as their distributions were skewed towards a few very large values. The matrix of environmental variables per plot initially included the same 12 variables used in the PCA. The variable vertical distance to the watercourse was log-transformed for the same reason. In order to reduce the environmental variables to the most significant ones, we performed a preliminary CCA with forward selection of environmental variables associated to Monte Carlo permutation tests for statistical significance. This procedure eliminated only the variable P. Additionally, we eliminated $\mathrm{K}^{+}, \mathrm{Ca}^{2+}$ and $\mathrm{Mg}^{2+}$ because they were strongly interrelated (high redundancy indicated by variance inflation factor $>20$ ) and best summarized by the synthetic variable SB. The remaining eight variables used in the final CCA were vertical distance to the watercourse, $\mathrm{pH}, \mathrm{Al}^{3+}, \mathrm{SB}$, organic matter, sand, silt and clay. The Monte Carlo permutation test (ter Braak, 1988) was performed to assess the significance of the correlations between the overall species abundance distributions and these eight environmental variables.

To examine the effects of environmental variables in particular species populations, we calculated Spearman's rank correlation coefficients, and their significance (Zar, 1996), between species abundances in the 18 plots and two chosen substrate variables. The species were the same 39 used in CCA and the environmental variables were SB and the vertical distance to the watercourse because they summarize soil chemical fertility and moisture, respectively.

\section{RESULTS}

\section{Environmental analysis}

The results of the PCA are shown in the ordination diagram prepared with the first two components (Fig. 3). The contributions of the first four components to the total variation, expressed by the eigenvalues, are $0.616,0.274,0.074$ and 0.029 , respectively. The proportional accumulated variances explained by these components are $61.9,89.0,96.4$ and $99.3 \%$. Therefore, the first two components summarize most variance contained in the environmental data. 


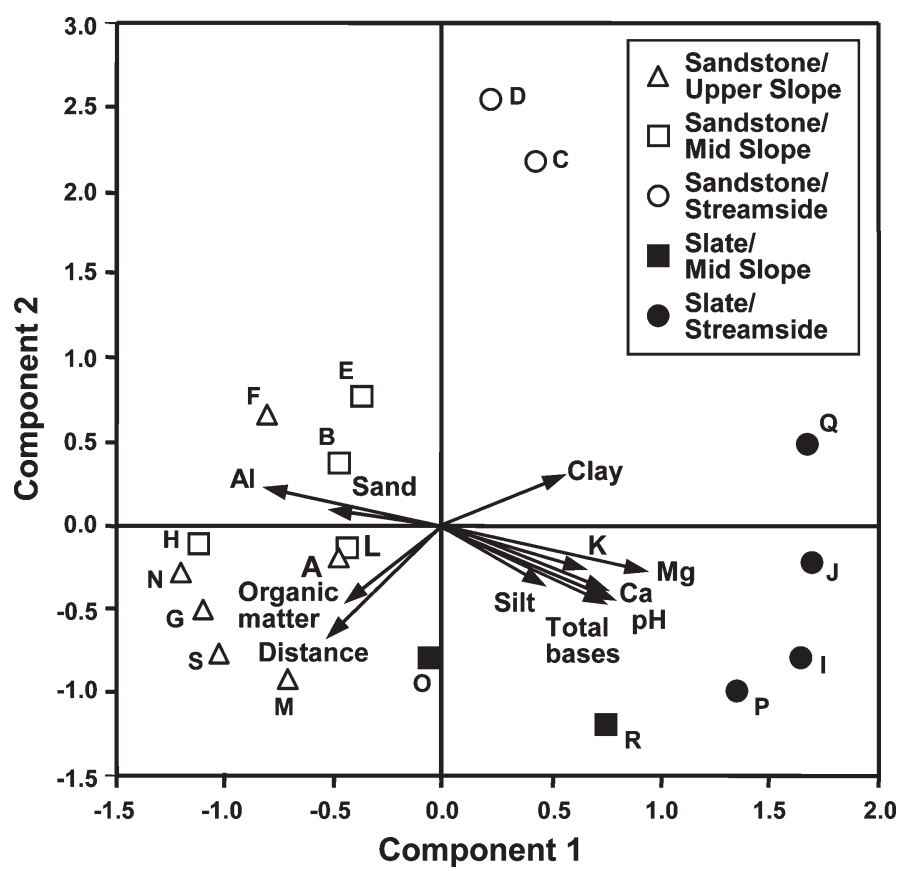

FIG. 3. Ordination diagram produced by principal components analysis (PCA) of 11 environmental variables (soil and topography) from the 18 sample plots in the valley forest of the Véu de Noiva waterfall. Each plot is identified by a letter (see Fig. 2) and given a particular symbol according to site as defined by underlying bedrock and topographic position. The arrows represent the main variation trend (direction and magnitude) of environmental variables. Distance $=$ vertical distance to the watercourse.

The first component separates the plots into two large groups, based essentially on soil fertility and texture, reflecting the two underlying bedrocks, sandstone and slate (Table 1). The Sandstone group is concentrated on the left side of the diagram and is strongly correlated with higher concentrations of $\mathrm{Al}^{3+}$ and proportions of sand. The Slate group, on the right side of the diagram, is strongly correlated with higher soil $\mathrm{pH}$, higher concentrations of $\mathrm{Ca}^{2+}, \mathrm{Mg}^{2+}, \mathrm{K}^{+}$and $\mathrm{SB}$, and higher proportions of silt and clay. The four plots of the Slate/Streamside sites are further from the centre of the diagram than those of the Mid Slope, suggesting internal variation among Slate plots based on fertility, distance to watercourse and organic matter. The second component separates the plots into groups corresponding to the topographic sectors and is strongly correlated with vertical distance to the watercourse and with soil organic matter. The two Sandstone/Streamside plots appear at the top of the diagram well away from the others probably because of their very low soil organic matter.

The comparison of soil variables among the five sites discriminated by the PCA indicates that most significant differences occur between the soils derived from either sandstone or slate (Table 1). In general, sandstone-derived soils are more 
TABLE 1. Environmental variables (1 topographic and 11 soil types) in the 18 sample plots. Figures are means \pm standard deviations of $N$ plots or soil samples collected in the five types of site defined by underlying bedrock and topographic position (US $=$ Upper Slope, $\mathrm{MS}=$ Mid Slope, $\mathrm{SS}=$ Streamside). Where $F$ tests rejected the null hypothesis $(P<0.05)$, means followed by different bold letters indicate significant differences in Tukey-Kramer tests $(P<0.05)$

\begin{tabular}{|c|c|c|c|c|c|c|c|}
\hline \multirow[b]{2}{*}{ Site } & \multirow[b]{2}{*}{$N$} & \multirow{2}{*}{$\begin{array}{l}\text { Vertical } \\
\text { distance }(\mathrm{m})\end{array}$} & \multirow{2}{*}{$\underline{\text { Sand }}$} & \multirow[t]{2}{*}{ Silt } & \multirow{2}{*}{$\frac{\text { Clay }}{(\%)}$} & Organic matter & \multirow[b]{2}{*}{$\mathrm{pH}\left(\mathrm{H}_{2} \mathrm{O}\right)$} \\
\hline & & & & & & & \\
\hline Sandstone/US & 6 & $38 \pm 16.3 \mathbf{a}$ & $75 \pm 4.7 \mathbf{a}$ & $17 \pm 3.9$ & $8 \pm 2.1 \mathbf{b}$ & $4.2 \pm 1.2 \mathbf{a}$ & $4.5 \pm 0.32 \mathbf{b}$ \\
\hline Sandstone/MS & 4 & $22 \pm 13.8 \mathbf{a b}$ & $72 \pm 5.7 \mathbf{a b}$ & $17 \pm 4.1$ & $11 \pm 2.5 \mathbf{a b}$ & $2.5 \pm 0.5 \mathbf{a b}$ & $4.2 \pm 0.10 \mathbf{b}$ \\
\hline Sandstone/SS & 2 & $4 \pm 0.7 \mathbf{b}$ & $70 \pm 5.7 \mathbf{a b}$ & $18 \pm 5.0$ & $12 \pm 0.7 \mathbf{a b}$ & $1.4 \pm 0.0 \mathbf{b}$ & $4.5 \pm 0.35 \mathbf{b}$ \\
\hline Slate/MS & 2 & $25 \pm 2.8 \mathbf{a b}$ & $60 \pm 8.5 \mathbf{b}$ & $27 \pm 6.6$ & $13 \pm 2.1 \mathrm{a}$ & $2.0 \pm 0.5 \mathbf{b}$ & $5.3 \pm 0.00 \mathrm{a}$ \\
\hline Slate/SS & 4 & $8 \pm 3.2 \mathbf{b}$ & $65 \pm 2.6 \mathbf{b}$ & $23 \pm 2.6$ & $12 \pm 0.0 \mathbf{a b}$ & $2.6 \pm 0.5 \mathbf{a b}$ & $5.5 \pm 0.19 \mathbf{a}$ \\
\hline \multirow[t]{3}{*}{ ANOVAS } & $F$ & 5.10 & 4.40 & 3.022 & 3.919 & 5.77 & 19.34 \\
\hline & $P$ & 0.011 & 0.018 & 0.054 & 0.027 & 0.067 & $<10^{-4}$ \\
\hline & \multirow[b]{2}{*}{$N$} & $\begin{array}{l}\text { Extractable } \\
\mathrm{P}\end{array}$ & $\mathrm{K}^{+}$ & $\mathrm{Ca}^{2+}$ & $\mathrm{Mg}^{2+}$ & $\mathrm{Al}^{3+}$ & $\begin{array}{l}\text { Sum of } \\
\text { bases }\end{array}$ \\
\hline Site & & \multicolumn{2}{|c|}{$\left(\mathrm{mg} \mathrm{dm}^{-3}\right)$} & \multicolumn{4}{|c|}{$\left(\mathrm{cmol}_{\mathrm{c}} \mathrm{dm}^{-3}\right)$} \\
\hline Sandstone/US & 6 & $5.8 \pm 1.72$ & $32 \pm 11.8 \mathbf{c}$ & $0.7 \pm 0.46 \mathbf{b}$ & $0.2 \pm 0.00 \mathbf{c}$ & $3.0 \pm 0.98 \mathbf{a}$ & $1.0 \pm 0.38 \mathbf{b}$ \\
\hline Sandstone/MS & 4 & $4.5 \pm 0.58$ & $41 \pm 18.7$ bc & $0.5 \pm 0.13 \mathbf{b}$ & $0.2 \pm 0.00 \mathbf{c}$ & $2.2 \pm 0.66 \mathbf{a b}$ & $0.8 \pm 0.17 \mathbf{b}$ \\
\hline Sandstone/SS & 2 & $4.0 \pm 1.41$ & $37 \pm 3.5 \mathbf{c}$ & $0.5 \pm 0.14 \mathbf{b}$ & $0.2 \pm 0.00 \mathbf{c}$ & $1.8 \pm 0.21 \mathbf{a b}$ & $0.8 \pm 0.14 \mathbf{b}$ \\
\hline Slate/MS & 2 & $6.0 \pm 0.00$ & $101 \pm 16.3 \mathbf{a}$ & $0.9 \pm 0.07 \mathbf{a b}$ & $0.6 \pm 0.42 \mathbf{b}$ & $0.9 \pm 0.64$ bc & $1.7 \pm 0.42 \mathbf{b}$ \\
\hline Slate/SS & 4 & $16.2 \pm 20.5$ & $66 \pm 12.8 \mathbf{a b}$ & $2.7 \pm 1.10 \mathbf{a}$ & $1.0 \pm 0.10 \mathrm{a}$ & $0.6 \pm 0.17 \mathrm{c}$ & $3.9 \pm 1.10 \mathbf{a}$ \\
\hline \multirow[t]{2}{*}{ ANOVAS } & $F$ & 1.47 & 11.42 & 2.22 & 29.41 & 8.53 & 17.30 \\
\hline & $P$ & 0.253 & $<10^{-3}$ & $<10^{-3}$ & $<10^{-5}$ & 0.013 & $<10^{-4}$ \\
\hline
\end{tabular}

acidic, contain higher amounts of exchangeable aluminium and lower amounts of exchangeable $\mathrm{K}^{+}, \mathrm{Ca}^{2+}$ and $\mathrm{Mg}^{2+}$, and are coarser textured than slate-derived soils. $\mathrm{SB}$ is significantly higher only for Slate/Streamside soils. There is a significant increase in the levels of $\mathrm{Al}^{3+}$ and organic matter with increasing elevation within the Sandstone group. The levels of extractable $\mathrm{P}$ and silt did not differ significantly among sites.

\section{Tree community}

The comparison of physiognomic variables of the tree community among the sites (Table 2) indicates that tree density in the Sandstone sites was higher at the Streamside and lower in the Mid and Upper Slope while basal area was higher in the Streamside and Mid Slope and lower in the Upper Slope. In the Slate sites, basal area was higher and density was lower in the Streamside than in the Mid Slope. Mean tree height within the Sandstone and Slate sites increased with decreasing 
TABLE 2. Physiognomic variables for the total sample and the five types of site defined by underlying bedrock and topographic position

\begin{tabular}{lccccccc}
\hline \hline Site & $\begin{array}{l}\text { No. of } \\
\text { plots }\end{array}$ & Area (ha) & $\begin{array}{l}\text { No. of Density } \\
\text { trees }\end{array}$ & $\begin{array}{l}\text { Basal area } \\
(\text { trees ha }\end{array}$ & $\begin{array}{l}\text { dbh* Height* } \\
\left(\mathrm{m}^{2} \mathrm{ha}^{-1}\right)\end{array}$ & $\begin{array}{l}\text { (cm) } \\
(\mathrm{m})\end{array}$ \\
\hline Sandstone/Upper Slope & 6 & 0.36 & 408 & 1133 & 20.825 & 11.8 & 8.2 \\
Sandstone/Mid Slope & 4 & 0.24 & 262 & 1092 & 26.550 & 13.5 & 9.3 \\
Sandstone/Streamside & 2 & 0.12 & 191 & 1592 & 25.400 & 11.6 & 9.8 \\
Slate/Mid Slope & 2 & 0.12 & 206 & 1717 & 23.100 & 11.0 & 7.8 \\
Slate/Streamside & 4 & 0.24 & 269 & 1121 & 24.829 & 12.7 & 8.4 \\
Total sample & 18 & 1.08 & 1336 & 1237 & 23.749 & 12.2 & 8.5 \\
\hline \hline
\end{tabular}

*Means.

elevation and the mean diameter was highest at the Sandstone/Mid Slope and Slate/ Streamside sites.

The 148 species registered in the sample plots with their quantitative parameters calculated for the total sample and the five sites defined by the PCA are given in Table 3. Among the 110 species sampled with more than one individual, Trichilia claussenii, Guarea kunthiana, Xylopia emarginata, Sebastiana membranifolia, Metrodorea stipularis, Mollinedia schottiana, Erythroxylum anguifugum and Piper amalago are exclusive to the Slate/Streamside plots. Tapirira guianensis, Vatairea macrocarpa, Qualea multiflora, Diptychandra aurantiaca, Myrciaria floribunda, Xylopia aromatica, Licania kunthiana, Vochysia haenkeana, Manihot tripartita, Ficus krukovii, Ocotea corymbosa and Cybianthus brasiliensis were found only in the Sandstone/Upper Slope plots. Myrsine lancifolia was found only in the Sandstone/ Mid Slope and Trichilia pallida in the Slate/Mid Slope. No species in that group was exclusive to the Sandstone/Streamside. The composition of the tree community is quite different among sites in terms of most abundant species. Of the 54 extracted from the top 15 of each site, only eight (14.8\%) are common to both Sandstone and Slate sites. Among the three Sandstone sites, nine species are common to the Upper and Mid Slope, four to the Mid Slope and Streamside, and only two (3.7\%) to the Upper Slope and Streamside. The two Slate sites share only four species. These results show that Slope and Streamside sites are quite distinct floristically for both the Sandstone and Slate areas.

\section{Species-environment relationships}

The results of the CCA are given in the ordination diagrams (Fig. 4) and the correlation matrix (Table 4). The eigenvalues of the first two CCA axes were 0.418 and 0.228 , respectively, indicating a moderately 'long' gradient for the first axis $(\sim 0.5)$, i.e. there is considerable species turnover along the gradient (ter Braak, 1995), and a 'short' gradient for the second axis $(\ll 0.5)$, i.e. most species occur throughout the gradient and vary essentially in abundance. CCA axes accounted for a low 
TABLE 3. Tree species recorded. Nt, number of trees; BA, total basal area; Rs, Spearman rank correlations with respective significance $(P)$ between the species abundance (number of trees) and two environmental variables: sum of bases and vertical distance to the watercourse ( $N=18$ plots). $P$-values $<0.05$ are underlined in bold. Species ranked by descending Nt. Correlations are given only for the 39 species with $\mathrm{Nt}>10$. (The suffix 'aceae' is omitted from family names.)

\begin{tabular}{|c|c|c|c|c|c|c|c|}
\hline \multirow[b]{2}{*}{ Species } & \multirow[b]{2}{*}{ Families } & \multirow[b]{2}{*}{$\mathrm{Nt}$} & \multirow{2}{*}{$\begin{array}{l}\mathrm{BA} \\
\left(\mathrm{m}^{2}\right)\end{array}$} & \multicolumn{4}{|c|}{ Sum of bases Vertical distance } \\
\hline & & & & $R s$ & $P$ & $R s$ & $P$ \\
\hline Siparuna guianensis Aublet & Siparun. & 88 & 0.310 & 0.02 & 0.952 & 0.10 & 0.682 \\
\hline Ecclinusa ramiflora Mart. & Sapot. & 65 & 0.994 & -0.02 & 0.950 & -0.23 & 0.350 \\
\hline $\begin{array}{l}\text { Calyptranthes paniculata } \\
\text { Ruiz \& Pav. }\end{array}$ & Myrt. & 50 & 0.486 & -0.22 & 0.370 & 0.49 & $\underline{0.038}$ \\
\hline $\begin{array}{l}\text { Protium guianense subsp. } \\
\text { pilosissimum (Engler) Daly }\end{array}$ & Burser. & 47 & 0.209 & -0.56 & $\underline{0.016}$ & -0.40 & 0.097 \\
\hline Unonopsis lindmanii R.E.Fries & Annon. & 45 & 0.534 & 0.44 & 0.071 & -0.18 & 0.486 \\
\hline Pseudolmedia laevigata Trécul & Mor. & 45 & 0.772 & -0.01 & 0.981 & -0.46 & $\underline{0.055}$ \\
\hline Sloanea tuerckheimii Donn. Sm. & Elaeocarp. & 45 & 1.300 & -0.35 & 0.154 & -0.30 & 0.222 \\
\hline Matayba guianensis Aublet & Sapind. & 42 & 0.421 & 0.27 & 0.270 & -0 & 0.096 \\
\hline Trichilia claussenii C.DC. & Meli. & 35 & 0.533 & 0.63 & $\underline{0.005}$ & -0 & 0.074 \\
\hline Eugenia florida DC. & Myrt. & 26 & & 0. & & -0 & $\underline{0.007}$ \\
\hline Inga vera Willd. & $F a b$. & 25 & 0.242 & -0.11 & 0.668 & & 0.127 \\
\hline $\begin{array}{l}\text { Protium heptaphyllum (Aublet) } \\
\text { Marchand }\end{array}$ & Burser. & 24 & 0.308 & 0.39 & 0.113 & 0.02 & 0.951 \\
\hline $\begin{array}{l}\text { Coussarea hydrangeifolia } \\
\text { (Benth.) Benth. \& Hook.f. }\end{array}$ & Rubi. & 23 & 0.171 & 0.61 & $\underline{0.008}$ & -0.21 & 0.398 \\
\hline Miconia chartacea Triana & Melastomat. & 21 & 0.145 & -0.32 & 0.189 & 0.27 & 0.280 \\
\hline Nectandra cuspidata Nees & Laur. & 21 & .510 & -0.22 & & & 0.543 \\
\hline Ormosia arborea (Vell.) Harms & $F a b$. & 20 & 0.229 & 0.13 & & & 0.270 \\
\hline $\begin{array}{l}\text { Sacoglottis mattogrossensis } \\
\text { Malme }\end{array}$ & Humiri. & 20 & 0.335 & -0.37 & 0.134 & 0.29 & 0.241 \\
\hline $\begin{array}{l}\text { Ocotea pomaderroides } \\
\text { (Meisn.) Mez }\end{array}$ & Laur. & 20 & 0.400 & -0.29 & 0.240 & 0.41 & 0.095 \\
\hline Guatteria conspicua R.E.Fries & & 19 & & -0.29 & & & 0.069 \\
\hline Miconi & Melas & 18 & & & & & 0.175 \\
\hline Miconia matthaei Naudin & Melastomat. & 18 & 0.081 & -0.50 & $\underline{0.035}$ & -0.52 & $\underline{0.028}$ \\
\hline $\begin{array}{l}\text { Bocageopsis mattogrossensis } \\
\text { R.E.Fries }\end{array}$ & Annon. & 18 & 0.269 & -0.53 & $\underline{0.023}$ & 0.56 & $\underline{0.015}$ \\
\hline $\begin{array}{l}\text { Cheiloclinium cognatum (Miers.) } \\
\text { A.C.Smith }\end{array}$ & Celastr. & 16 & 0.132 & 0.27 & 0.270 & -0.47 & $\underline{0.048}$ \\
\hline Cecropia pachystachya Trécul & Urtic. & 16 & & & 0.134 & -0.09 & 0.722 \\
\hline Ocotea aciphylla (Nees) Mez & Laur. & 14 & 1.081 & -0.47 & $\underline{0.049}$ & & $\underline{0.043}$ \\
\hline Tocoyena brasiliensis Mart. & Rubi. & 13 & 0.062 & 0.24 & $\overline{0.339}$ & -0.23 & $\overline{0.361}$ \\
\hline Miconia punctata (Desr.) D.Don & Melastomat. & 13 & 0.158 & 0.16 & 0.524 & -0.47 & $\underline{0.047}$ \\
\hline Maprounea guianensis Aublet & Euphorbi. & 13 & 0.200 & -0.50 & $\underline{0.034}$ & 0.05 & 0.836 \\
\hline $\begin{array}{l}\text { Chrysophyllum amazonicum } \\
\text { T.D.Penn. }\end{array}$ & Sapot. & 13 & 0.229 & 0.27 & $\overline{0.272}$ & 0.20 & 0.420 \\
\hline Tapirira guianensis Aublet & Anacardi. & 13 & 0.265 & 0.02 & & & $\underline{0.039}$ \\
\hline Oenocarpus distichus Mart. & Arec. & 13 & 0.493 & -0.16 & 0.528 & 0.44 & 0.066 \\
\hline
\end{tabular}


TABLE 3. (Cont'd).

\begin{tabular}{|c|c|c|c|c|c|c|c|}
\hline \multirow[b]{2}{*}{ Species } & \multirow[b]{2}{*}{ Families } & \multirow[b]{2}{*}{$\mathrm{Nt}$} & \multirow{2}{*}{$\begin{array}{l}\text { BA } \\
\left(\mathrm{m}^{2}\right)\end{array}$} & \multicolumn{4}{|c|}{ Sum of bases Vertical distance } \\
\hline & & & & $R s$ & $P$ & $R s$ & $P$ \\
\hline Sclerolobium paniculatum Benth. & Fab. & 13 & 0.587 & -0.09 & 0.711 & 0.32 & 0.195 \\
\hline Hymenaea courbaril L. & $F a b$. & 13 & 0.843 & 0.09 & 0.720 & -0.30 & 0.220 \\
\hline $\begin{array}{l}\text { Physocalymma scaberrimum } \\
\text { Pohl }\end{array}$ & Lythr. & 12 & 0.169 & -0.13 & 0.611 & 0.20 & 0.436 \\
\hline Machaerium brasiliense Vogel & $F a b$. & 12 & 0.279 & 0.36 & 0.142 & 0.05 & 0.836 \\
\hline Pouteria ramiflora Radlk. & Sapot. & 12 & 0.513 & -0.25 & 0.324 & 0.18 & 0.469 \\
\hline $\begin{array}{l}\text { Jacaranda copaia (Aublet) } \\
\text { D.Don. }\end{array}$ & Bignoni. & 12 & 1.009 & -0.60 & $\underline{0.009}$ & -0.25 & 0.314 \\
\hline $\begin{array}{l}\text { Sapium glandulosum } \\
\text { (L.) Morong }\end{array}$ & Euphorbi. & 11 & 0.189 & 0.50 & $\underline{0.036}$ & -0.24 & 0.336 \\
\hline Ocotea elegans $\mathrm{Mez}$ & Laur. & 10 & 0.459 & -0.29 & 0.236 & -0.05 & 0.836 \\
\hline Inga cayennensis Sagot & $F a b$. & 9 & 0.022 & - & - & - & - \\
\hline Qualea multiflora Mart. & Vochysi. & 9 & 0.039 & - & - & - & - \\
\hline Miconia longifolia (Aublet) DC. & Melastomat. & 9 & 0.070 & - & - & - & - \\
\hline $\begin{array}{l}\text { Licania apetala (E.Meyer) } \\
\text { Fritsch }\end{array}$ & Chrysobalan. & 9 & 0.197 & - & - & - & - \\
\hline $\begin{array}{l}\text { Priogymnanthus hasslerianus } \\
\text { (Chodat) P.S.Green }\end{array}$ & Ole. & 9 & 0.305 & - & - & - & - \\
\hline $\begin{array}{l}\text { Micropholis venulosa } \\
\quad \text { (Mart. \& Eichler) Pierre }\end{array}$ & Sapot. & 9 & 0.903 & - & - & - & - \\
\hline $\begin{array}{l}\text { Casearia arborea } \\
\text { (L.C.Rich.) Urban }\end{array}$ & Salic. & 8 & 0.077 & - & - & - & - \\
\hline $\begin{array}{l}\text { Sebastiania membranifolia } \\
\text { Müll.Arg. }\end{array}$ & Euphorbi. & 8 & 0.101 & - & - & - & - \\
\hline Matayba elaeagnoides Radlk. & Sapind. & 8 & 0.103 & - & - & - & - \\
\hline Guarea guidonia (L.) Sleumer & Meli. & 8 & 0.132 & - & - & - & - \\
\hline Quiina rhitidopus Tul. & Quiin. & 8 & 0.177 & - & - & - & - \\
\hline $\begin{array}{l}\text { Protium spruceanum } \\
\text { (Benth.) Engler }\end{array}$ & Burser. & 8 & 0.201 & - & - & - & - \\
\hline $\begin{array}{l}\text { Alchornea glandulosa } \\
\text { Poepp. \& Endl. }\end{array}$ & Euphorbi. & 8 & 0.410 & - & - & - & - \\
\hline $\begin{array}{l}\text { Connarus perrottetii } \\
\text { (DC.) Planchon }\end{array}$ & Connar. & 7 & 0.052 & - & - & - & - \\
\hline Virola sebifera Aublet & Myristic. & 7 & 0.075 & - & - & - & - \\
\hline Amaioua guianensis Aublet & Rubi. & 7 & 0.091 & - & - & - & - \\
\hline $\begin{array}{l}\text { Miconia splendens } \\
\quad \text { (Swartz) Griseb. }\end{array}$ & Melastomat. & 7 & 0.091 & - & - & - & - \\
\hline $\begin{array}{l}\text { Elaeoluma glabrescens } \\
\text { (Mart. \& Eichler) Pierre }\end{array}$ & Sapot. & 7 & 0.358 & - & - & - & - \\
\hline $\begin{array}{l}\text { Apuleia leiocarpa } \\
\text { (Vogel) Macbr. }\end{array}$ & $F a b$. & 7 & 0.491 & - & - & - & - \\
\hline $\begin{array}{l}\text { Sloanea guianensis } \\
\text { (Aublet) Benth. }\end{array}$ & Elaeocarp. & 7 & 0.619 & - & - & - & - \\
\hline $\begin{array}{l}\text { Bellucia grossularioides } \\
\text { (L.) Triana }\end{array}$ & Melastomat. & 6 & 0.030 & - & - & - & - \\
\hline
\end{tabular}


TABle 3. (Cont'd).

\begin{tabular}{|c|c|c|c|c|c|c|c|}
\hline \multirow[b]{2}{*}{ Species } & \multirow[b]{2}{*}{ Families } & \multirow[b]{2}{*}{$\mathrm{Nt}$} & \multirow{2}{*}{$\begin{array}{l}\text { BA } \\
\left(\mathrm{m}^{2}\right)\end{array}$} & \multicolumn{4}{|c|}{ Sum of bases Vertical distance } \\
\hline & & & & $R s$ & $P$ & $R s$ & $P$ \\
\hline Tetragastris cerradicola Daly & Burser. & 6 & 0.099 & - & - & - & - \\
\hline Inga marginata Willd. & $F a b$. & 6 & 0.102 & - & - & - & - \\
\hline Guarea kunthiana A.Juss. & Meli. & 6 & 0.139 & - & - & - & - \\
\hline $\begin{array}{l}\text { Chrysophyllum gonocarpum } \\
\text { (Mart. \& Eichler) Engler }\end{array}$ & Sapot. & 6 & 0.161 & - & - & - & - \\
\hline Cordia bicolor A.DC. & Boragin. & 6 & 0.372 & - & - & - & - \\
\hline Alibertia macrophylla K.Schum. & Rubi. & 5 & 0.018 & - & - & - & - \\
\hline $\begin{array}{l}\text { Licania sclerophylla } \\
\text { (Mart.) Fritsch }\end{array}$ & Chrysobalan. & 5 & 0.023 & - & - & - & - \\
\hline Piper arboreum Aublet & Piper. & 5 & 0.023 & - & - & - & - \\
\hline $\begin{array}{l}\text { Allophylus edulis (A.St.-Hil.) } \\
\text { Radlk. }\end{array}$ & Sapind. & 5 & 0.025 & - & - & - & - \\
\hline Urera baccifera (L.) Gaud. & Urtic. & 5 & 0.046 & - & - & - & - \\
\hline Licania hoehnei Pilger & Chrysobalan. & 5 & 0.055 & - & - & - & - \\
\hline $\begin{array}{l}\text { Bauhinia longifolia (Bongard) } \\
\text { D.Dietrich }\end{array}$ & $F a b$. & 5 & 0.072 & - & - & - & - \\
\hline Heisteria ovata Benth. & Olac. & 5 & 0.120 & - & - & - & - \\
\hline Spondias mombin L. & Anacardi. & 5 & 0.215 & - & - & - & - \\
\hline Copaifera langsdorffii Desf. & Fab. & 5 & 0.301 & - & - & - & - \\
\hline $\begin{array}{l}\text { Sparattosperma leucanthum } \\
\text { (Vell.) K.Schum. }\end{array}$ & Bignoni. & 5 & 0.813 & - & - & - & - \\
\hline Sorocea guilleminiana Gaudich. & Mor. & 4 & 0.013 & - & - & - & - \\
\hline Xylopia aromatica (Lam.) Mart. & Annon. & 4 & 0.027 & - & - & - & - \\
\hline Myrsine lancifolia Mart. & Myrsin. & 4 & 0.028 & - & - & - & - \\
\hline $\begin{array}{l}\text { Myrciaria floribunda } \\
\text { (Willd.) O.Berg }\end{array}$ & Myrt. & 4 & 0.029 & - & - & - & - \\
\hline Hirtella glandulosa Sprengel & Chrysobalan. & 4 & 0.085 & - & - & - & - \\
\hline Mabea fistulifera Mart. & Euphorbi. & 4 & 0.108 & - & - & - & - \\
\hline $\begin{array}{l}\text { Cybianthus brasiliensis } \\
\text { (Mez) Agostini }\end{array}$ & Myrsin. & 3 & 0.006 & - & - & - & - \\
\hline Vismia guianensis (Aublet) Pers. & Clusi. & 3 & 0.008 & - & - & - & - \\
\hline Miconia nervosa (Smith) Triana & Melastomat. & 3 & 0.009 & - & - & - & - \\
\hline Manihot tripartita Müll.Arg. & Euphorbi. & 3 & 0.010 & - & - & - & - \\
\hline Casearia gossypiosperma Briquet & Salic. & 3 & 0.014 & - & - & - & - \\
\hline $\begin{array}{l}\text { Buchenavia capitata } \\
\text { (Vahl) Eichler }\end{array}$ & Combret. & 3 & 0.020 & - & - & - & - \\
\hline Licania kunthiana Hook.f. & Chrysobalan. & 3 & 0.022 & - & - & - & - \\
\hline $\begin{array}{l}\text { Himatanthus lancifolius } \\
\text { (Müll.Arg.) Woodson }\end{array}$ & Apocyn. & 3 & 0.039 & - & - & - & - \\
\hline $\begin{array}{l}\text { Tabebuia serratifolia } \\
\text { (Vahl) Nichols }\end{array}$ & Bignoni. & 3 & 0.041 & - & - & - & - \\
\hline $\begin{array}{l}\text { Schefflera morototoni } \\
\text { (Aublet) Maguire, } \\
\text { Steyerm. \& Frodin }\end{array}$ & Arali. & 3 & 0.094 & - & - & - & - \\
\hline Metrodorea stipularis Mart. & Rut. & 3 & 0.106 & - & - & - & - \\
\hline
\end{tabular}


TABLE 3. (Cont'd).

\begin{tabular}{|c|c|c|c|c|c|c|c|}
\hline \multirow[b]{2}{*}{ Species } & \multirow[b]{2}{*}{ Families } & \multirow[b]{2}{*}{$\mathrm{Nt}$} & \multirow{2}{*}{$\begin{array}{l}\text { BA } \\
\left(\mathrm{m}^{2}\right)\end{array}$} & \multicolumn{4}{|c|}{ Sum of bases Vertical distance } \\
\hline & & & & $R s$ & $P$ & $R s$ & $P$ \\
\hline Diptychandra aurantiaca Tul. & $F a b$. & 3 & 0.132 & - & - & - & - \\
\hline Xylopia emarginata Mart. & Annon. & 3 & 0.241 & - & - & - & - \\
\hline $\begin{array}{l}\text { Vatairea macrocarpa } \\
\text { (Benth.) Ducke }\end{array}$ & Fab. & 3 & 0.260 & - & - & - & - \\
\hline Erythroxylum anguifugum Mart. & Erythroxyl. & 2 & 0.004 & - & - & - & - \\
\hline Ocotea corymbosa (Meisn.) Mez & Laur. & 2 & 0.006 & - & - & - & - \\
\hline $\begin{array}{l}\text { Mollinedia schottiana } \\
\text { (Sprengel) Perkins }\end{array}$ & Monimi. & 2 & 0.007 & - & - & - & - \\
\hline Piper amalago $\mathrm{L}$. & Piper. & 2 & 0.007 & - & - & - & - \\
\hline Ficus krukovii Standley & Mor. & 2 & 0.009 & - & - & - & - \\
\hline Trichilia pallida Swartz & Meli. & 2 & 0.010 & - & - & - & - \\
\hline Duguetia echinophora R.E.Fries & Annon. & 2 & 0.020 & - & - & - & - \\
\hline Vochysia haenkeana Mart. & Vochysi. & 2 & 0.032 & - & - & - & - \\
\hline $\begin{array}{l}\text { Gomidesia hebepetala } \\
\text { (DC.) O.Berg }\end{array}$ & Myrt. & 2 & 0.038 & - & - & - & - \\
\hline Calyptranthes strigipes O.Berg & Myrt. & 2 & 0.039 & - & - & - & - \\
\hline Aspidosperma spruceanum Benth. & Apocyn. & 2 & 0.080 & - & - & - & - \\
\hline $\begin{array}{l}\text { Sterculia apetala (Jacquin) } \\
\text { H.Karst. }\end{array}$ & Malv. & 2 & 0.121 & - & - & - & - \\
\hline Pera glabrata (Schott) Poepp. & Euphorbi. & 2 & 0.127 & - & - & - & - \\
\hline Attalea phalerata Mart. & Arec. & 2 & 0.186 & - & - & - & - \\
\hline Aegiphila triantha Schauer & Lami. & 1 & 0.002 & - & - & - & - \\
\hline $\begin{array}{l}\text { Cybianthus guyanensis } \\
\text { (A.DC.) Miq. }\end{array}$ & Myrsin. & 1 & 0.002 & - & - & - & - \\
\hline Eugenia uniflora L. & Myrt. & 1 & 0.002 & - & - & - & - \\
\hline $\begin{array}{l}\text { Heteropterys byrsonimifolia } \\
\text { A.Juss. }\end{array}$ & Malpighi. & 1 & 0.002 & - & - & - & - \\
\hline Inga heterophylla Willd. & $F a b$. & 1 & 0.002 & - & - & - & - \\
\hline Mouriri acutiflora Naudin & Memecyl. & 1 & 0.002 & - & - & - & - \\
\hline $\begin{array}{l}\text { Rhamnidium elaeocarpum } \\
\text { Reissek }\end{array}$ & Rhamn. & 1 & 0.002 & - & - & - & - \\
\hline $\begin{array}{l}\text { Schefflera vinosa } \\
\text { (Cham. \& Schltdl.) } \\
\text { Frodin \& Fiaschi }\end{array}$ & Arali. & 1 & 0.002 & - & - & - & - \\
\hline Banara tomentosa Clos & Salic. & 1 & 0.003 & - & - & - & - \\
\hline $\begin{array}{l}\text { Endlicheria paniculata } \\
\text { (Sprengel) Macbr. }\end{array}$ & Laur. & 1 & 0.003 & - & - & - & - \\
\hline Heisteria densifrons Engler & Olac. & 1 & 0.003 & - & - & - & - \\
\hline Roupala brasiliensis Klotzsch & Prote. & 1 & 0.003 & - & - & - & - \\
\hline Aegiphila lhotskiana Cham. & Lami. & 1 & 0.004 & - & - & - & - \\
\hline Apeiba tibourbou Aublet & Malv. & 1 & 0.004 & - & - & - & - \\
\hline $\begin{array}{l}\text { Ceiba speciosa (A.St.-Hil.) } \\
\text { Ravenna }\end{array}$ & Malv. & 1 & 0.004 & - & - & - & - \\
\hline $\begin{array}{l}\text { Miconia minutiflora } \\
\text { (Bonpl.) DC. }\end{array}$ & Melastomat. & 1 & 0.004 & - & - & - & - \\
\hline
\end{tabular}


TABLE 3. (Cont'd).

\begin{tabular}{|c|c|c|c|c|c|c|c|}
\hline \multirow[b]{2}{*}{ Species } & \multirow[b]{2}{*}{ Families } & \multirow[b]{2}{*}{$\mathrm{Nt}$} & \multirow{2}{*}{$\begin{array}{l}\text { BA } \\
\left(\mathrm{m}^{2}\right)\end{array}$} & \multicolumn{4}{|c|}{ Sum of bases Vertical distance } \\
\hline & & & & $R s$ & $P$ & $R s$ & $P$ \\
\hline Ziziphus mistol Griseb. & Rhamn. & 1 & 0.004 & - & - & - & - \\
\hline $\begin{array}{l}\text { Chloroleucon tortum } \\
\text { (Mart.) Pittier }\end{array}$ & $F a b$. & 1 & 0.005 & - & - & - & - \\
\hline Virola albidiflora Ducke & Myristic. & 1 & 0.005 & - & - & - & - \\
\hline Astrocaryum aculeatum Meyer & Arec. & 1 & 0.006 & - & - & - & - \\
\hline Eupatorium laeve DC. & Aster. & 1 & 0.006 & - & - & - & - \\
\hline Ficus insipida Willd. & Mor. & 1 & 0.006 & - & - & - & - \\
\hline $\begin{array}{l}\text { Hyeronima alchorneoides } \\
\text { Fr.Allem. }\end{array}$ & Phyllanth. & 1 & 0.006 & - & - & - & - \\
\hline Acacia polyphylla DC. & $F a b$. & 1 & 0.007 & - & - & - & - \\
\hline Ficus maxima P.Miller & Mor. & 1 & 0.007 & - & - & - & - \\
\hline Cybianthus cuneifolius Mart. & Myrsin. & 1 & 0.008 & - & - & - & - \\
\hline Terminalia glabrescens Mart. & Combret. & 1 & 0.009 & - & - & - & - \\
\hline Astronium fraxinifolium Schott & Anacardi. & 1 & 0.011 & - & - & - & - \\
\hline Byrsonima coriacea (Swartz) DC. & Malpighi. & 1 & 0.015 & - & - & - & - \\
\hline Maclura tinctoria (L.) D.Don. & Mor. & 1 & 0.019 & - & - & - & - \\
\hline Styrax pohlii A.DC. & Styrac. & 1 & 0.026 & - & - & - & - \\
\hline Antonia ovata Pohl & Logani. & 1 & 0.027 & - & - & - & - \\
\hline Myrcia tomentosa (Aublet) DC. & Myrt. & 1 & 0.048 & - & - & - & - \\
\hline Guazuma ulmifolia Lam. & Malv. & 1 & 0.078 & - & - & - & - \\
\hline Emmotum nitens (Benth.) Miers & Icacin. & 1 & 0.088 & - & - & - & - \\
\hline Poecilanthe parviflora Benth. & $F a b$. & 1 & 0.115 & - & - & - & - \\
\hline Simarouba amara Aublet & Simaroub. & 1 & 0.126 & - & - & - & - \\
\hline $\begin{array}{l}\text { Eriotheca gracilipes } \\
\text { (K.Schum.) A.Robyns }\end{array}$ & Malv. & 1 & 0.255 & - & - & - & - \\
\hline Totals: 148 & 52 & 1336 & 25.66 & & & & \\
\hline
\end{tabular}

cumulative percentage variance of species abundance data, $20.1 \%$ and $31.0 \%$ for axes 1 and 2, respectively, indicating that considerable 'noise' remained unexplained. However, low percentage variances for species data are normal in vegetation data and do not impair the significance of species-environment relations (ter Braak, 1988). In fact, CCA produced quite high values for both species-environment correlations (axis $1=0.97$, axis $2=0.93$ ). In addition, the Monte Carlo permutation test demonstrated that the species abundances and environmental variables were significantly correlated $(F$-ratio $=2.74, P<0.01)$.

The two underlying bedrocks appear as very distinct clusters of plots in the CCA diagram (Fig. 4A). The first canonical axis is most strongly (and negatively) correlated with $\mathrm{Al}$, followed by sum of bases and $\mathrm{pH}$ (both positively), sand (negatively), and silt, clay and organic matter (all positively) (Table 4). These soil variables are also highly interrelated (see weighted correlation matrix in Table 4). The second canonical axis is more strongly influenced by the vertical distance to the watercourse while the other seven variables yielded very low correlations. The elevational 
A

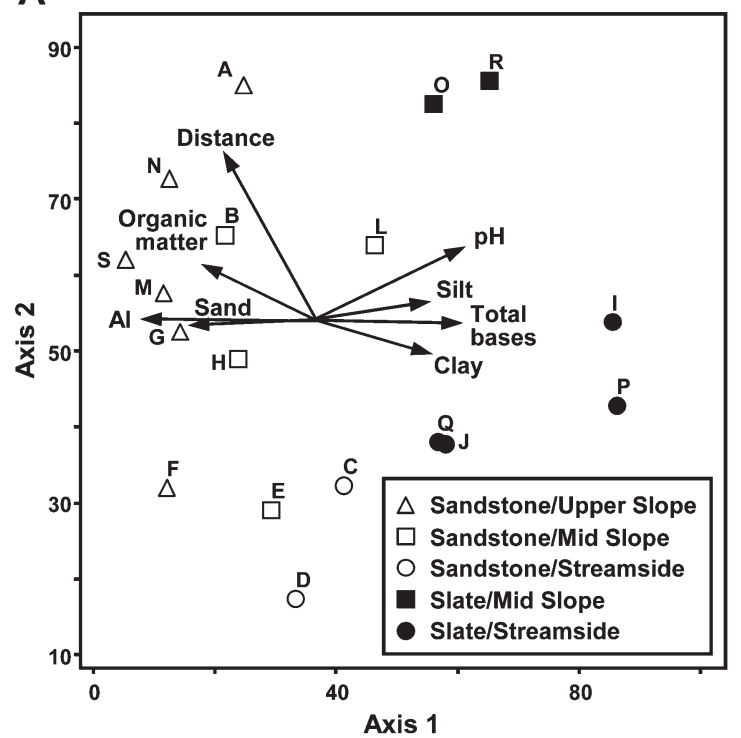

B

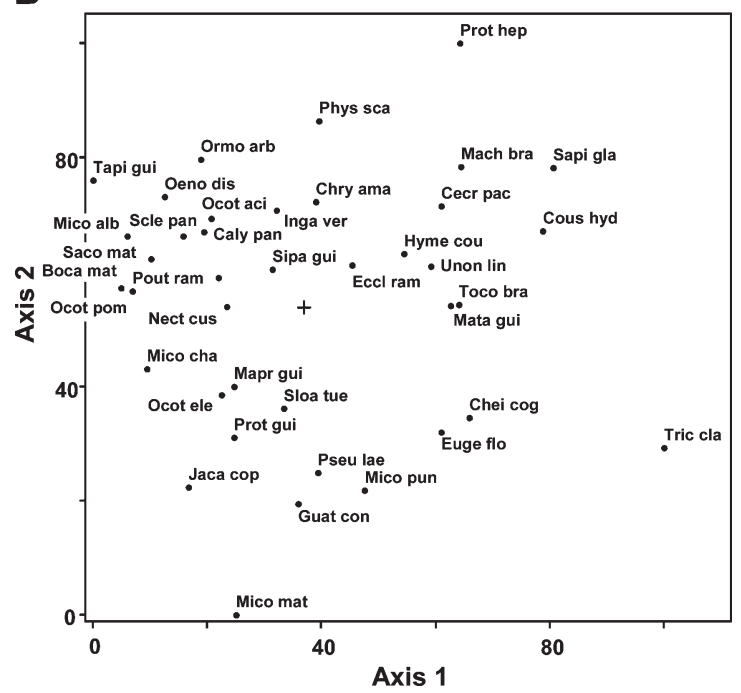

FIG. 4. Ordination diagrams produced by canonical correspondence analysis (CCA) of the number of individuals of the 39 most abundant species $(N \geq 10)$ sampled in eighteen $600 \mathrm{~m}^{2}$ plots in the valley forest of the Véu de Noiva waterfall, and their correlation with environmental variables. Ordination of plots and environmental variables (A) and ordination of species (B) on the first two CCA axes are given in separate diagrams for clarity. Each plot is identified by a letter (see Fig. 2) and given a particular symbol according to site as defined by underlying bedrock and topographic position. The arrows represent the main variation trend (direction and magnitude) of environmental variables. Distance $=$ vertical distance to the watercourse. Full species names are given in Table 3. 
TABLE 4. Canonical correspondence analysis (CCA): intraset correlations in the first two ordination axes, and matrix of weighted correlations for the eight environmental variables supplied. Distance = vertical distance to the watercourse. Correlations with absolute values $>0.5$ are underlined in bold

\begin{tabular}{|c|c|c|c|c|c|c|c|c|c|c|}
\hline \multirow[b]{2}{*}{$\begin{array}{l}\text { Environmental } \\
\text { variables }\end{array}$} & \multicolumn{2}{|c|}{ Intraset correlations } & \multicolumn{8}{|c|}{ Environmental variables } \\
\hline & Axis 1 & Axis 2 & Distance & $\mathrm{pH}$ & $\mathrm{Al}$ & $\begin{array}{l}\text { Sum of } \\
\text { bases }\end{array}$ & $\begin{array}{l}\text { Organic } \\
\text { matter }\end{array}$ & Sand & Silt & Clay \\
\hline Distance & -0.48 & $\underline{0.76}$ & - & & & & & & & \\
\hline $\mathrm{pH}$ & $\underline{0.75}$ & 0.32 & 0.14 & - & & & & & & \\
\hline $\mathrm{Al}$ & $-\underline{0.89}$ & 0.01 & $\underline{0.50}$ & $-\underline{0.56}$ & - & & & & & \\
\hline Sum of bases & $\underline{0.77}$ & -0.01 & 0.26 & $\underline{0.80}$ & -0.49 & - & & & & \\
\hline Organic matter & $-\underline{0.56}$ & 0.26 & $\underline{0.66}$ & -0.07 & $\underline{0.75}$ & 0.03 & - & & & \\
\hline Sand & $-\underline{0.66}$ & -0.01 & 0.29 & $-\underline{0.50}$ & 0.39 & -0.45 & 0.26 & - & & \\
\hline Silt & $\underline{0.58}$ & 0.08 & 0.08 & $\underline{0.53}$ & -0.26 & $\underline{0.50}$ & -0.02 & $-\underline{0.95}$ & - & \\
\hline Clay & $\underline{0.57}$ & -0.14 & $\underline{0.62}$ & 0.22 & $-\underline{0.52}$ & 0.15 & $-\underline{0.68}$ & $-\underline{0.71}$ & 0.45 & - \\
\hline
\end{tabular}

gradient appears as a diagonal in the CCA diagram (Fig. 4A). These trends are also highly correlated with the differences in soil properties among sites given in Table 1.

The species ordination by CCA (Fig. 4B) indicates correlation with the environmental variables and therefore suggests their preference for the five habitats defined by soil fertility and texture and topography. For instance, Trichilia claussenii, Cheiloclinium cognatum, Eugenia florida, Matayba guianensis and Coussarea hydrangeifolia show a preference for the Slate/Streamside site, while Protium heptaphyllum, Cecropia pachystachya, Sapium glandulosum, Machaerium brasiliense, Tocoyena brasiliensis, Unonopsis lindmanii, Hymenaea courbaril and Ecclinusa ramiflora prefer the Slate/Mid Slope sites. Physocalymma scaberrimum, Chrysophyllum amazonicum, Inga vera, Ocotea aciphylla, Calyptranthes paniculata, Sclerolobium paniculatum, Siparuna guianensis, Pouteria ramiflora, Nectandra cuspidata, Maprounea guianensis, Sloanea tuerckheimii, Ocotea elegans, Protium guianense subsp. pilosissimum and Jacaranda copaia show a preference for the Sandstone/Mid Slope sites. Miconia punctata, Pseudolmedia laevigata, Guatteria conspicua and Miconia matthaei seem to prefer the Sandstone/Streamside, and Tapirira guianensis, Miconia albicans, Oenocarpus distichus, Ormosia arborea, Sacoglottis mattogrossensis, Bocageopsis mattogrossensis, Ocotea pomaderroides and Miconia chartacea, the Sandstone/Upper Slope sites.

The species ordination by CCA (Fig. 4B) strongly agrees with their correlation coefficients with the two chosen substrate variables (Table 3), vertical distance to the watercourse and total bases. Of the 39 species involved in the analysis, 12 showed significant correlations with either of the two variables and three with both variables. The three species with positively significant correlations with total bases, Trichilia claussenii, Coussarea hydrangeifolia and Sapium glandulosum, also appeared on the left side of the ordination diagram. In contrast, the six species 
with negative correlations with total bases, Protium guianense subsp. pilosissimum, Miconia matthaei, Bocageopsis mattogrossensis, Ocotea aciphylla, Maprounea guianenis and Jacaranda copaia, appeared on the right side of the diagram. The four species with positively significant correlations with vertical distance to the watercourse, Calyptranthes paniculata, Bocageopsis mattogrossensis, Ocotea aciphylla and Tapirira guianensis, are all concentrated in the upper part of the diagram, whilst the five negatively correlated with vertical distance, Pseudolmedia laevigata, Eugenia florida, Miconia matthaei, M. punctata and Cheiloclinium cognatum, are concentrated at the bottom.

\section{DISCUSSION}

Catenary variations of soil properties in tropical forests have commonly been attributed to topography itself, because variations in steepness and different topographical levels on a slope may determine, to a great extent, different ground-water regimes and these, in turn, usually affect soil texture and nutritional status (Bourgeron, 1983; Newbery \& Proctor, 1984; Furley, 1992; Silva-Júnior, 1998; Silva Júnior et al., 1996; Oliveira-Filho et al., 1997, 2001; van den Berg \& Oliveira-Filho, 1999). This was our initial model for studying the tree community gradients in this area. However, an unexpected pattern related to soil origin was revealed in the analyses and proved to be the leading factor influencing the distribution of tree species in the forest. A gradient related to topographic levels was also shown but was less pronounced. Both the environmental analysis (PCA) and the speciesenvironment analysis (CCA) produced similar results and indicated that five sites could be defined for the valley forest based on underlying bedrock and topographic site.

The influence of the local bedrocks in pedogenesis certainly caused the strong variation in soil texture and nutritional status within the canyon. Siltstones, slates, and other poor, pellitic rocks in Brazil are normally poor in Ca but rich in $\mathrm{K}$ and give rise to fine-textured soils; sandstones, on the other hand, often produce very dystrophic and coarse-textured soils (Resende et al., 1995). Previous studies have demonstrated that the strong dichotomy between soils originating from either slate or sandstone in the region of the Chapada dos Guimarães plateau and Baixada Cuiabana lowlands is reflected in the composition of the woody plant communities of both arboreal savannas ('cerradão') (Oliveira-Filho \& Martins, 1986, 1991) and gallery forests (Oliveira-Filho et al., 1990). In both cases, the authors identified soil chemical fertility and water storage capacity as the main factors determining differences in species composition. In the case of arboreal savannas, soil fertility is the chief factor resulting in two well-known physiognomies found throughout Central Brazil: the 'mesotrophic cerradão' and the 'dystrophic cerradão' (Ratter, 1971, 1992; Ratter et al., 1973, 1978, 2003). Soil fertility was found to be the leading factor underlying the differentiation between adjacent evergreen forest on dystrophic soils and semideciduous forest on mesotrophic soils (Ratter et al., 1973). 
In addition to differences in soil fertility, the two bedrocks give rise to soils with rather different water regimes. Sandstone-derived soils are much coarser and, therefore, have stronger drainage and lower water storage capacity than slate-derived soils (Resende et al., 1988). It is quite difficult to separate the effects of groundwater and soil fertility on the differentiation of the plant communities growing on either soil. The importance of ground-water regime in species distribution in the valley forest is reinforced by the second level gradient dictated by the topographic levels. A single soil water gradient for the valley forest sites can be deduced, from highest to lowest water availability:

\section{Slate/Streamside $\rightarrow$ Sandstone/Streamside $\rightarrow$ Slate/Mid Slope $\rightarrow$ Sandstone/Mid Slope $\rightarrow$ Sandstone/Upper Slope}

Habitat differentiation also influenced tree community physiognomy. The concentration of large trees in the Sandstone/Mid Slope, Sandstone/Streamside and Slate/ Streamside sites may be due to stronger competition for light in the lower parts of the canyon and to higher water availability (Oliveira-Filho et al., 2001). Mean tree stature is less in upper sites, perhaps due to lower nutrient and water availability factors that may restrict tree growth (Lleras \& Kirkbride, 1978). The higher density of smaller trees in the Slate/Mid Slope sites is probably related to soil depth, since the slate bedrock outcrops or is near the surface over the whole site. In these conditions, root systems are shallow, resulting in poor stability of the larger trees, which frequently fall forming gaps that provide conditions for the establishment of many small individuals.

The present study is of an exploratory nature and the indication of habitat preferences by particular species can only be confirmed after repeated studies or experimental work. For some species, there is already published evidence that the patterns shown are repeated elsewhere. Examples are the association of Pseudolmedia laevigata and Cheiloclinium cognatum with moister soils (Oliveira-Filho \& Ratter, 1995) and Trichilia claussenii with richer soils (Oliveira-Filho et al., 1997). For some species however there is always the possibility that environmental variables not directly related to the substrate may be the actual leading factors. Light regime is the major candidate through both gap-phase dynamics and light gradients caused by forest physiognomic variations (Denslow, 1987). The higher abundance of the pioneer tree Cecropia pachystachya in the Slate/Mid Slope, for example, probably results from the higher gap formation rate in those sites, as discussed above, and not from local soil fertility and moisture. In addition, canopy openness visibly increased away from the valley bottom and this certainly affects tree establishment in the understorey. Because light and topography gradients are related in this case, it is very difficult to differentiate their effects on plant distribution. The higher abundance of Miconia albicans in the Sandstone/Upper Slope sites, for example, may be due both to its preference for strongly drained sites and to its light-demanding behaviour (Espírito-Santo et al., 2002). To conclude, there is strong evidence that variations in the substrate, particularly soil moisture and fertility, were indeed important factors influencing tree species distribution in the valley forest. On the 
other hand, there was evidence that other factors, particularly light, may also be involved. Great care should be taken when interpreting apparent substrate-species relationships.

\section{ACKNOWLEDGEMENTS}

We are grateful to Marco Aurélio Leite Fontes (DCF/UFLA) and Eduardo van den Berg (DBI/UFLA), for their critical reading of the manuscript, to Libério Amorim Neto (IB/UFMT), who helped in the identification of the plant specimens, and to the students of the Forestry Program of FENF/UFMT, especially those of the Clube do Tereré, who helped with the field work. The first and second authors received research grants from CNPq (Proc. $n^{\circ} 135485 / 95-9$ and 301644/88-8, respectively).

\section{REFERENCES}

Askew, G. P., Moffatt, D. J., Montgomery, R. F. \& Searl, P. S. (1971). Soils and soil moisture as factors influencing the distribution of the vegetation formations of the Serra do Roncador, Mato Grosso. In: FerR I, M. G. (ed.) III Simpósio sobre o Cerrado, pp. 150-160. São Paulo, Brazil: University of São Paulo.

Botrel, R. T., Oliveira-Filho, A. T., Rodrigues, L. A. \& Curi, N. (2002). Composição florística e estrutura da comunidade arbórea de um fragmento de floresta estacional semidecidual em Ingaí, MG, e a influência de variáveis ambientais na distribuição das espécies. Revista Brasil. Bot. 25: 195-213.

Bourgeron, P. S. (1983). Spatial aspects of vegetation structure. In: Golley, F. B. (ed.) Ecosystems of the World 14A - Tropical Rain Forest Ecosystems, Structure and Function, pp. 29-47. Amsterdam, Netherlands: Elsevier.

Clark, D. B., Clark, D. A. \& ReAd, J. M. (1998). Edaphic variation and the mesoscale distribution of tree species in a neotropical rain forest. J. Ecol. 86: 101-112.

Denslow, J. S. (1987). Tropical rainforest gaps and tree species diversity. Annu. Rev. Ecol. Syst. 18: 431-451.

Eiten, G. (1975). The vegetation of the Serra do Roncador. Biotropica 7: 112-135.

Eiten, G. (1983). Classificação da vegetação do Brasil. Brasília, Brazil: Conselho Nacional de Desenvolvimento Científico e Tecnológico.

Eiten, G. (1994). Vegetação do cerrado. In: Pinto, M. N. (ed.) Cerrado-caracterização, ocupação e perspectives, pp. 17-73. Brasília, Brazil: University of Brasília.

EMBR APA (1997). Manual de métodos de análises de solo, 2nd edition. Rio de Janeiro, Brazil: Empresa Brasileira de Pesquisa Agropecuária, Centro Nacional de Pesquisa de Solos.

Espírito-Santo, F. D. B., Oliveira-Filho, A. T., Machado, E. L. M., Souza, J. S., Fontes, M. A. L. \& Marques, J. J. G. S. M. (2002). Variáveis ambientais e a distribuição de espécies arbóreas em um remanescente de floresta estacional semidecídua montana no campus da Universidade Federal de Lavras, MG. Acta Bot. Brasil. 16: 331-356.

FELfili, J. M. (1995). Diversity, structure and dynamics of a gallery forest in Central Brazil. Vegetatio 117: 1-15.

FOWLER, N. (1988). The effects of environmental heterogeneity in space and time on the regulation of populations and communities. In: DAvy, A. J., Hutchings, M. J. \& Watkinson, A. R. (eds) Plant Population Ecology, pp. 249-269. Oxford: Blackwell. 
Furley, P. A. (1992). Notes on the Soils and Plant Communities of Fazenda Agua Limpa (Brasília, D.F., Brazil). Occasional Publications No. 5. Edinburgh: University of Edinburgh.

HAll, R. B. W. \& HARCombe, P. A. (1998). Flooding alters apparent position of floodplain saplings on a light gradient. Ecology 79: 847-855.

IBA MA (1994). Plano de ação emergencial: documento de informações básicas do Parque Nacional da Chapada dos Guimarães. Cuiabá, Brazil: Instituto Brasileiro do Meio Ambiente e dos Recursos Naturais Renováveis, Delegacia Estadual de Mato Grosso.

Kent, M. \& Coker, P. (1992). Vegetation Description and Analysis: A Practical Approach. London: Belhaven.

Lleras, E. \& Kirk BRide, J. H. (1978). Alguns aspectos da vegetação da Serra do Cachimbo. Acta Amazonica 1/2: 51-65.

Macedo, R. L. G. \& Pinto, J. R. R. (1996). O perfil do visitante do Parque Nacional da Chapada dos Guimarães. In: Anais do $4^{\circ}$ Simpósio Internacional sobre Ecossistemas Florestais - FOREST 96, pp. 114-116. Belo Horizonte, Brazil: Sociedade de Investigação Florestal - SIF.

Marimon, B. S., Felfili, J. M. \& Lima, E. S. (2002). Floristics and phytosociology of the gallery forest of the Bacaba stream, Nova Xavantina, Mato Grosso, Brazil. Edinburgh J. Bot. 59: 303-318.

Metzger, J. P., Bernacci, L. C. \& GoldenberG, R. (1997). Pattern of tree species diversity in riparian forest fragments of different widths (SE Brazil). Plant Ecol. 133: $135-152$.

MMA (1982). Projeto Radambrasil: folhas SE 21 - Corumbá e SD.21 - Chapada dos Guimarães; geomorfologia, pedologia; vegetação e uso potencial da terra. Brasília, Brazil: Ministério das Minas e Energia.

Newbery, D. McC. \& Proctor, J. (1984). Ecological studies in four contrasting lowland rain forests in Gunung Mulu National Park, Sarawak. IV. Association between tree distribution and soil factors. J. Ecol. 72: 475-493.

Oliveira-Filho, A. T., Curi, N., Vilela, E. A. \& Carvalho, D. A. (1997). Tree species distribution along soil catenas in riverside semideciduous forest in southeastern Brazil. Flora 192: 47-64.

Oliveira-Filho, A. T., Curi, N., Vilela, E. A. \& Carvalho, D. A. (2001). Variation in tree community composition and structure with changes in soil properties within a fragment of semideciduous forest in south-eastern Brazil. Edinburgh J. Bot. 58: $139-158$.

Oliveir A-Filho, A. T. \& Martins, F. R. (1986). Distribuição, caracterização e composição florística das formações vegetais da região da Salgadeira, na Chapada do Guimarães (MT). Revista Brasil. Bot. 9: 207-223.

Oliveira-Filho, A. T. \& Martins, F. R. (1991). A comparative study of five cerrado areas in southern Mato Grosso, Brazil. Edinburgh J. Bot. 48: 307-322.

Oliveira-Filho, A. T. \& Ratter, J. A. (1995). A study of the origin of central Brazilian forests by the analysis of plant species distribution patterns. Edinburgh J. Bot. 52: 141-194.

Oliveira-Filho, A. T. \& Ratter, J. A. (2002). Vegetation physiognomies and woody flora of the Cerrado Biome. In: Oliveira, P. S. \& MARquis, R. J. (eds) The Cerrados of Brazil: Ecology and Natural History of a Neotropical Savanna, pp. 91-120. New York: Columbia University Press.

Oliveira-Filho, A. T., Ratter, J. A. \& Shepherd, G. J. (1990). Floristic composition and community structure of a Central Brasilian gallery forest. Flora 184: 103-117. 
Oliveira-Filho, A. T., Vilela, E. A., Gavilanes, M. L. \& Carvalho, D. A. (1994). Effect of flooding regime and understory bamboos on the physiognomy and tree species composition of a tropical semideciduous forest in southeastern Brazil. Vegetatio 113: 99-124.

RAtTER, J. A. (1971). Some notes on two types of cerradão occurring in northeastern Mato Grosso. In: FerR I, M. G. (ed.) III Simpósio sobre o Cerrado, pp. 100-102. São Paulo, Brazil: EDUSP.

RAtTer, J. A. (1992). Transitions between cerrado and forest vegetation in Brazil. In: Furley, P. A., Proctor, J. \& Ratter, J. A. (eds) Nature and Dynamics of Forest-Savanna Boundaries, pp. 417-429. London: Chapman \& Hall.

Ratter, J. A., Askew, G. P., Montgomery, R. F. \& Gifford, D. R. (1978). Observations on the vegetation of northeastern Mato Grosso, II. Forest and soils of the Rio Suiá-Missu area. Proc. Roy. Soc. London B 203: 191-208.

Ratter, J. A., Bridgewater, S. \& Ribeiro, J. F. (2003). Analysis of the floristic composition of the Brazilian cerrado vegetation of 376 areas. Edinburgh J. Bot. 60: $57-109$.

Ratter, J. A., Richards, P. W., Argent, G. \& Gifford, D. R. (1973). Observations on the vegetation of northeastern Mato Grosso, I. The woody vegetation types of the Xavantina - Cachimbo Expedition area. Phil. Trans. Roy. Soc. London B 226: 449-492.

Resende, M., Curi, N., Rezende, S. B. \& CorrêA, G. F. (1995). Pedologia: base para distinção de ambientes. Viçosa, Brazil: Núcleo de Estudo de Planejamento e Uso da Terra.

Resende, M., Curi, N. \& Santana, D. P. (1988). Pedologia e fertilidade do solo: interações e interpretações. Brasília, Brazil: Ministério da Agricultura.

Ribeiro, J. F. \& Walter, B. M. T. (1998). Fitofisionomias do Bioma Cerrado. In: Sano, S. M. \& Almeida, S. P. (eds) Cerrado: ambiente e flora, pp. 87-166. Planaltina, Brazil: EMBRAPA-CPAC.

Rodrigues, R. R. \& NAve, A. G. (2000). Heterogeneidade florística das matas ciliares. In: Rodrigues, R. R. \& Leitão-Filho, H. F. (eds) Matas ciliares: conservação e recuperação, pp. 45-71. São Paulo, Brazil: EDUSP.

RodRigues, R. R. \& SHEPhERD, G. J. (2000). Fatores condicionantes da vegetação ciliar. In: Rodrigues, R. R. \& Leitão-Filho, H. F. (eds) Matas ciliares: conservação e recuperação, pp. 101-107. São Paulo, Brazil: EDUSP.

Rosales, J., Petts, G. \& Knab-Vispo, C. (2001). Ecological gradients within the riparian forests of the lower Caura River, Venezuela. Plant Ecol. 152: 101-118.

Schiavini, I. (1997). Environmental characterization and groups of species in gallery forests. In: Proceedings of the International Symposium on Assessment and Monitoring of Forests in Tropical Dry Regions with Special Reference to Gallery Forests, pp. 107-113. Brasília, Brazil: University of Brasília.

Silva Júnior, M. C., Furley, P. A. \& Ratter, J. A. (1996). Variation in the tree communities and soils with slope in gallery forest, Federal District, Brazil. In: Anderson, M. G. \& Brooks, S. M. (eds) Advances in Hillslope Processes, vol. 1, pp. 451-469. London: John Wiley \& Sons.

Silva-Júnior, M. C. (1998). Comunidades de árvores e sua relação com os solos na Mata do Pitoco, Reserva Ecológica do IBGE, Brasília - DF. Revista Árv. 22: 29-40.

Ter borgh, J. (1992). Diversity and the Tropical Rain Forest. New York: Scientific American Library.

TER BRAAK, C. J. F. (1987). The analysis of vegetation-environment relationships by canonical correspondence analysis. Vegetatio 69: 69-77. 
TER BRAAK, C. J. F. (1988). CANOCO - A FORTRAN program for canonical community ordination by (Partial) (Detrended) (Canonical) correspondence analysis, principal components analysis and redundancy analysis, version 2.1. Technical report LWA-88-2. Wageningen, Netherlands: TNO - Institute of Applied Computer Science.

TER BraAk, C. J. F. (1995). Ordination. In: Jongman, R. H. G., Ter BraAk, C. J. F. \& van Tongeren, O. F. R. (eds) Data Analysis in Community and Landscape Ecology, pp. 91-173. Cambridge: Cambridge University Press.

TER BraAk, C. J. F. \& Šmilauer, P. (1998). CANOCO Reference Manual and User's Guide to CANOCO for Windows: Software for Canonical Community Ordination (version 4). Ithaca, NY: Microcomputer Power.

VAn den Berg, E. \& Oliveira-Filho, A. T. (1999). Spatial partitioning among tree species within an area of tropical montane gallery forest in south-eastern Brazil. Flora 194: 249-266.

ZAR, J. K. (1996). Biostatistical Analysis. New Jersey: Prentice-Hall.

Received 20 August 2003; accepted after minor revision 20 September 2005 